\title{
Germ-line deletion in DICER 1 revealed by a novel MLPA assay using synthetic oligonucleotides
}

\author{
Nelly Sabbaghian ${ }^{1,2}$, Archana Srivastava ${ }^{1,2}$, Nancy Hamel ${ }^{1,3}$, François Plourde ${ }^{1,2}$, Malgorzata Gajtko-Metera ${ }^{4}$, \\ Marek Niedziela ${ }^{5}$ and William D Foulkes ${ }^{\star, 1,2,3}$
}

DICER1 is an endoribonuclease responsible for the production of mature microRNAs which are small, single-stranded RNA molecules that regulate gene expression post-transcriptionally by binding to mRNA and repressing the expression of target genes. Germ-line mutations in DICER1 are responsible for a rare cancer syndrome, including tumors that can co-occur with multinodular goiter (MNG). Using Sanger sequencing, we screened all DICER1 exons and intron boundaries in 20 suspected mutation carriers: nine with ovarian sex cord-stromal tumors (including Sertoli-Leydig cell tumors (SLCTs)), five with pleuropulmonary blastoma, one with cystic nephroma, one with nasal chondromesenchymal hamartoma and four with more than one manifestation suggestive of a germ-line DICER1 mutation. All were negative for any apparently deleterious variants. We developed a Multiplex Ligation-based Probe Amplification assay for DICER1 to screen for large deletions or duplications. Synthetic oligonucleotides were designed to cover all exons in three probe-mixes. In a child with a SLCT and MNG, and in her mother and brother (both diagnosed with $\mathrm{MNG}$ ), we identified a heterozygous germ-line deletion of approximately 3 kilobases that eliminates exon 21 of DICER1 and two-thirds of intron 21, accompanied by an insertion of a Gucleotide at the $3^{\prime}$ end of the deletion (c.3270-6_4051-1280delinsG). This allele is expressed in the patient's cDNA, creating an out-of-frame deletion predicted to result in a truncated protein (r.3270_4050del; p.Tyr1091Ser*28). Our novel finding of a disease-causing large deletion in DICER1 emphasizes the need to include assays that can detect rearrangements, duplications and deletions in any DICER1 screening protocol. European Journal of Human Genetics (2014) 22, 564-567; doi:10.1038/ejhg.2013.215; published online 25 September 2013

Keywords: DICER1; Multiplex Ligation-based Probe Amplification (MLPA); Sertoli-Leydig cell tumor (SLCT); synthetic oligos; probe-mixes; multinodular goiter; miRNA

\section{INTRODUCTION}

Germ-line mutations in the RNase III endonuclease DICER1 were first identified in children diagnosed with pleuropulmonary blastoma, ${ }^{1}$ a rare lung tumor that arises in children aged $<72$ months. ${ }^{2}$ Subsequently, germ-line mutations have been reported in $\sim 50$ children, adolescents and young adults with cystic nephroma, ovarian sex cord-stromal tumor (especially the Sertoli-Leydig cell tumor (SLCT) sub-type), multinodular goiter (MNG), embryonal rhabomyosarcoma, Ewing's tumor of the cervix, infratentorial and supratentorial primitive neuroepithelial tumors, Wilms tumor, ciliary body medulloepithelioma and pituitary blastoma. ${ }^{1,3-8}$ Thus far, all deleterious mutations identified have been located within exons or close to intron-exon boundaries. Numerous studies in other Mendelian syndromes have indicated that a varying proportion of deleterious mutations in the causative genes are attributable to mutations that delete or rearrange whole exons, either individually or multiply. Multiplex ligation-dependent probe amplification (MLPA) was first applied to identifying deletions, duplications or rearrangements affecting the breast cancer susceptibility gene $B R C A 1^{9}$ and has become the most widely used technique for identifying such largerscale alterations. ${ }^{10}$ In some populations, over one-quarter of all
BRCA1 mutations are caused by these types of mutations, ${ }^{9}$ and in others a third of all mismatch repair gene mutations are accounted for by exonic deletions. ${ }^{11}$ Thus their contribution to the overall proportion of mutations in a gene can be substantial. The principle behind MLPA involves designing oligonucleotides (oligos) that hybridize to specific sequences in target genes. Two juxtaposed oligos designed to also contain PCR primer-binding sequences form the probe that gets amplified by PCR. Successful amplification of the probe by PCR will occur if juxtaposed oligos each bind to their target sequence adjacent to each other such that they can be ligated together (Supplementary Figure S1). The amount of probe amplified is then quantified; if the DNA sample tested lacks part or all of the target sequence on one allele, or contains more than two copies of the target sequence, the number of probes that are amplified by PCR will be reduced or augmented compared with normal diploid controls. Thus one can find a deletion or duplication as a consequence of chromosomal rearrangements. ${ }^{9}$ No MLPA test is commercially available for DICER1 from MRC-Holland. ${ }^{12}$ Therefore, with the help of MRC-Holland, we developed an 'in-house' MLPA assay using synthetic oligos covering all the coding exons of DICER1 to screen persons who were negative for coding sequence mutations in

${ }^{1}$ Program in Cancer Genetics, Departments of Oncology and Human Genetics, McGill University, Montreal, Quebec, Canada; ${ }^{2}$ Lady Davis Institute, Segal Cancer Centre, Jewish General Hospital, Montreal, Quebec, Canada; ${ }^{3}$ The Research Institute, McGill University Health Centre, Montreal, Quebec, Canada; ${ }^{4}$ Department of Nuclear Medicine and Out-Patient Pediatric Endocrine Clinic, Children's Memorial Health Institute, Warsaw, Poland; ${ }^{5}$ Department of Pediatric Endocrinology and Rheumatology, Poznan University of Medical Sciences, Poznan, Poland

*Correspondence: Dr WD Foulkes, Program in Cancer Genetics, Departments of Oncology and Human Genetics, McGill University, Montreal, QC H2W 1S6, Canada. Tel: +514 9341934 × 44121; E-mail: William.foulkes@mcgill.ca

Received 22 April 2013; revised 30 July 2013; accepted 22 August 2013; published online 25 September 2013 
Table 1 Cases subjected to MLPA

\begin{tabular}{|c|c|c|c|}
\hline Diagnosis & $\begin{array}{l}\text { Age at diagnosis }(d x), \\
\text { (mo= months, } y=\text { years) }\end{array}$ & Relevant medical history & $\begin{array}{l}\text { Family history (possibly DICER1-related clinical } \\
\text { manifestations) }\end{array}$ \\
\hline \multicolumn{4}{|l|}{ Pleuropulmonary blastoma (PPB) } \\
\hline \multirow[t]{2}{*}{ PPB type I } & $2 \mathrm{mo}$ & No relevant medical history & No other affected family member \\
\hline & $1 \mathrm{y}$ & No relevant medical history & Maternal grandmother with hypothyroidism \\
\hline \multirow[t]{3}{*}{ PPB type III } & $4 y$ & No relevant medical history & $\begin{array}{l}\text { Paternal uncle with thyroid cyst in childhood and } \\
\text { colon polyps at } 30 \mathrm{y}\end{array}$ \\
\hline & $11 \mathrm{mo}$ & No relevant medical history & No other affected family member \\
\hline & $2 y$ & No relevant medical history & No other affected family member \\
\hline \multirow[t]{9}{*}{ Ovarian sex cord-stromal tumor (including SLCT) } & 35 y & No relevant medical history & No other affected family member \\
\hline & 55 y & No relevant medical history & $\begin{array}{l}\text { Sister with differentiated thyroid cancer at } 51 \text { y } \\
\text { and Leydig cell tumour at } 55 \text { y }\end{array}$ \\
\hline & $6 y$ & No relevant medical history & No other affected family member \\
\hline & $16 y$ & No relevant medical history & $\begin{array}{l}\text { Maternal uncle with enlarged thyroid, maternal } \\
\text { cousin with 'congenital kidney problems', maternal } \\
\text { aunt with 'ovarian cancer before childbearing' }\end{array}$ \\
\hline & 15 y & No relevant medical history & Maternal first cousin with brain tumour dx 6 y \\
\hline & $12 \mathrm{mo}$ & No relevant medical history & No other affected family member \\
\hline & 3 y & Autism spectrum disorder & No other affected family member \\
\hline & 46 y & Papillary thyroid cancer dx 46 y & No other affected family member \\
\hline & 35 y & $\begin{array}{l}\text { Burkitt lymphoma dx } 18 \mathrm{mo} \\
\text { pituitary insufficiency, iris } \\
\text { melanoma }\end{array}$ & No other affected family member \\
\hline Cystic nephroma (CN) & $2 y$ & No relevant medical history & $\begin{array}{l}\text { Paternal grandmother with 'hypothyroidism', } \\
\text { paternal great-grandmother with 'goiter in her } 40 \mathrm{~s} \text { 'a }\end{array}$ \\
\hline Nasal chondromesenchymal hamartoma (NCMH) & 9 y & No relevant medical history & No information \\
\hline \multirow[t]{4}{*}{$>1$ feature suggestive of DICER 1 mutation } & $24 y$ & MNG and SLCT & $\begin{array}{l}\text { One child with 'spina bifida', another child with } \\
\text { 'neuroblastoma'; father with 'MNG', paternal uncle } \\
\text { with 'MNG', paternal uncle with 'spina bifida' }\end{array}$ \\
\hline & $\begin{array}{l}5 \text { y } \mathrm{PPB} \text { and } 6 \text { y intestinal } \\
\text { polyps, } \mathrm{CN} \text {, and } \mathrm{NCMH}\end{array}$ & As stated & No other affected family member \\
\hline & $\begin{array}{l}\text { Birth and } 9 \text { mo PPB, } 3 \mathrm{mo} \\
\text { jejunal polyposis, } 6 \text { y } \mathrm{NCMH}\end{array}$ & As stated & No other affected family member \\
\hline & 6 y SLCT and 14 y MNG ${ }^{b}$ & As stated & $\begin{array}{l}\text { Mother with MNG (2 surgeries) and brother with } \\
\text { MNG dx } 15 \text { y }\end{array}$ \\
\hline
\end{tabular}

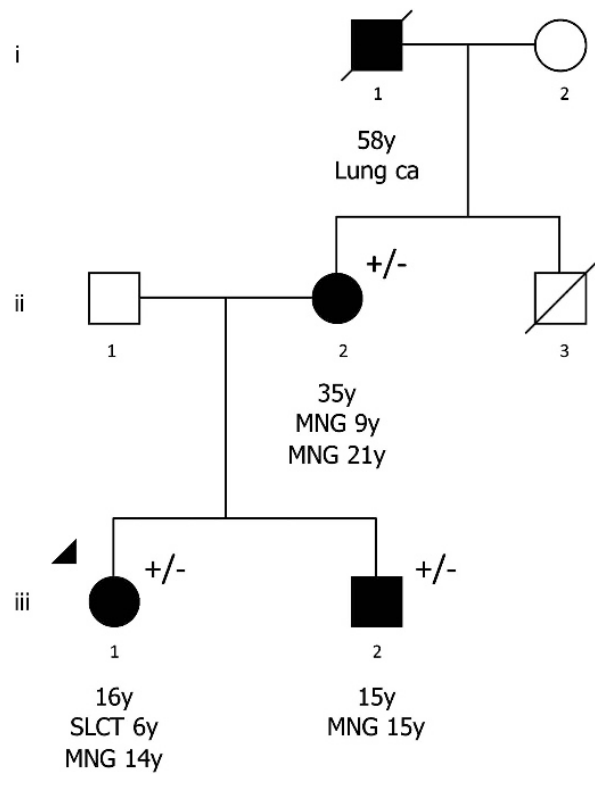

Figure 1 Pedigree of the proband with the exon 21 DICER1 deletion. Current age in years $(y)$ is shown below the symbol $(O$ : women; $\square$ : men; -10: known diagnosis). The arrowhead indicates the proband. $(+/-)$ indicates heterozygous status of the mutation. ii-2 required two separate operative removals of her MNG; at age $9 \mathrm{y}$, she had a partial left lobectomy of the thyroid, despite thyroid hormone replacement therapy, new lesions developed in both lobes were noted from age 13 y onwards and at age 21 y she underwent a total thyroidectomy. She was also diagnosed with a uterine leiomyoma at age $35 \mathrm{y}$.

DICER1 (NM_177438.2) when tested by Sanger sequencing. As subjects, we focused on a series of persons $(n=20$, Table 1$)$ for whom we estimated, based on published data, the probability of DICER1 mutations to be $>10 \%,{ }^{1,3-8}$ yet no likely deleterious coding sequence variants had been identified in DICER1 by Sanger sequencing. We report here the first characterized exonic deletion in DICER1.

\section{MATERIALS AND METHODS}

\section{Patient recruitment}

All patients and their families provided written informed consents to take part in the study, using consent forms approved by the relevant institutional review boards. 
a

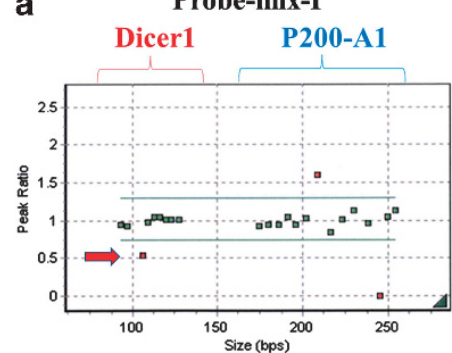

b

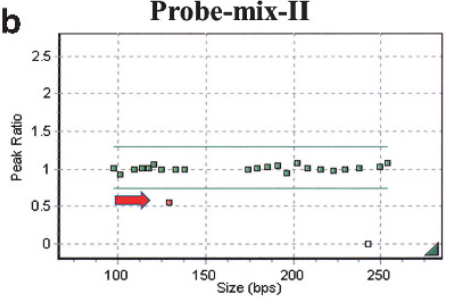

C

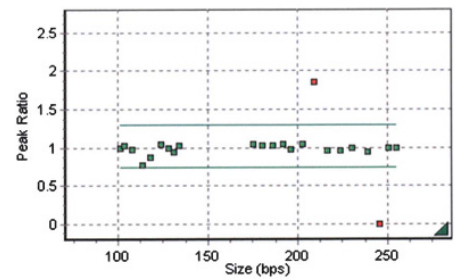

d

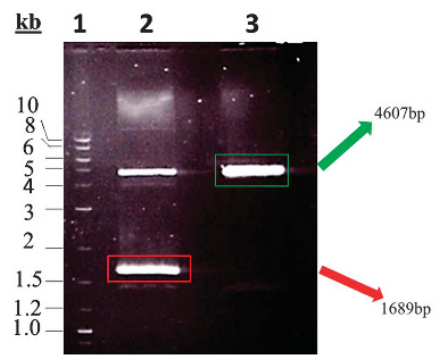

e c.3270-6_4051-1280delinsG

1

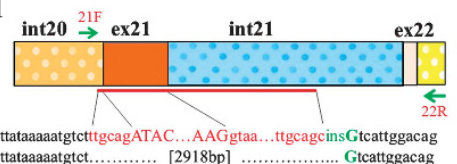

ttataaaaatgtct............ [2918bp] …............... Gtcattggacag

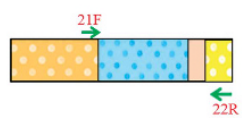

f
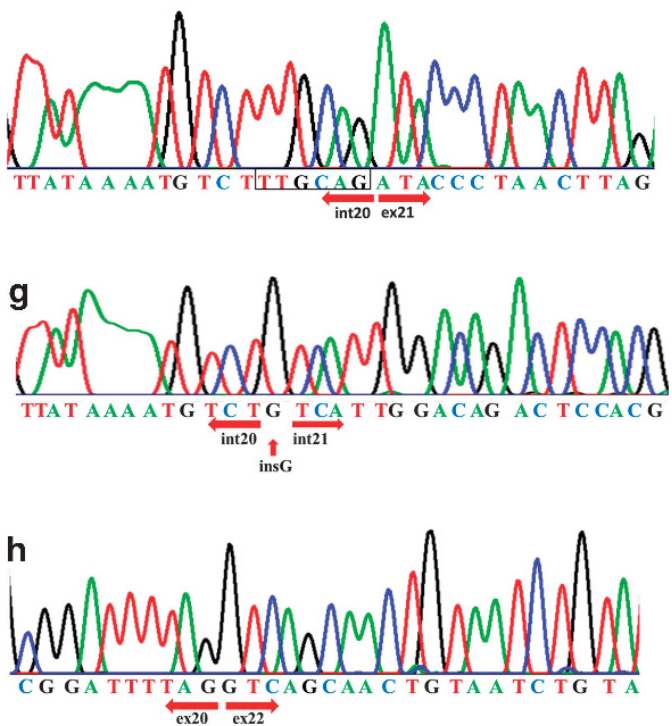

Figure 2 DICER1 MLPA and deletion validation. (a, band $\mathbf{c}$ ) Representation of the MLPA results using probe-mixes I, II and III, respectively, for the proband. Panels (a-c) were generated with GeneMarker v. 1.70 from SoftGenetics, LLC, (http://www.softgenetics.com/GeneMarker.html). The arrows point to the deletion. In a, the parentheses show the peak ratio values for the DICER1 probes and those for P200-A1 (control and reference probes from MRCHolland). (d) $0.8 \%$ agarose gel showing in lane 1 the 2 -log DNA ladder (NEB, Mississauga, Canada), lane 2 the two PCR fragments amplified in the proband to map the genomic deletion (e-1 $=4607 \mathrm{bp}$ and $\mathrm{e}-2=1689 \mathrm{bp}$ ) and lane 3 is the PCR fragment of the control (e-1). (e) Graphic representation of the two PCR fragments shown in panel d. (e-1) shows the wild-type DICER1 sequence (large PCR fragment) from the beginning of the deleted sequence in intron 20, TTGCAG, until the breakpoint in intron 21, followed by an insertion of a 'G' nucleotide; brown with white dots: intron 21; solid brown: exon 21; blue: intron 21; pink: exon 22 and yellow: intron 22. (e-2) The shorter fragment of the proband showing intron 20 next to intron 21 . (f) Sequencing trace of the control sample (e-1) showing the junction between intron 20 and exon 21. (g) Sequencing trace of the shorter PCR fragment (e-2) using primers 21F and $22 \mathrm{R}$ (same primers used for the control sample). One arrow points at the ' $G$ ' insertion and the two other arrows point in the direction of intron 20 and intron 21 showing the lack of exon 21. (h) Sequencing trace of the cDNA of the proband showing the junction between exon 20 and 22 (arrows) with the absence of exon 21 (r.3270_4050del).

\section{DNA, RNA extraction and cDNA synthesis}

For details about DNA, RNA extractions as well as cDNA synthesis and amplification, please refer to the Supplementary Material (Supplementary Methods section).

\section{MLPA assay and synthetic probe design}

Allele ID version $7.7^{13}$ from PREMIER Biosoft (Palo Alto, CA, USA) is the only software that designs synthetic probes for MLPA. It was originally developed with the collaboration of MRC-Holland (Amsterdam, Holland) to design assays for species identification by aligning the sequences and analyzing the conserved regions. MRC-Holland does not recommend exceeding 11 pairs of oligos in a probe-mix, and the difference in length between each amplified probe should be equal or superior to four nucleotides so they can be clearly separated on an ABI sequencer and analyzed with fragment analysis software. We designed three probe-mixes for DICER1 (Supplementary Table S1), and each DNA sample was tested in three MLPA reactions. The left and right hybridizing sequences (Supplementary Figure S1) were synthesized with the forward and reverse primer binding sequences at their $5^{\prime}$ and $3^{\prime}$ end, respectively, by Integrated DNA Technologies (IDT, Coralville, IA, USA). Full details of the design of the MLPA probes are provided in the Supplementary Methods, the synthetic hybridizing sequences for each exon are shown in
Supplementary Table S1 and the synthetic DICER1 and P200-A1 probes and sizes are shown in Supplementary Table S2.

MLPA assay protocol details are supplied in Supplementary Methods.

\section{Agarose gel electrophoresis and Sanger sequencing}

Fragments from long-range PCR and cDNA amplification were separated by agarose gel electrophoresis and sequenced (details in Supplementary Methods).

\section{Mutation nomenclature and public access}

We used the Mutalyzer web-based program (https://mutalyzer.nl/) to verify the correct HGVS nomenclature for the large deletion. The reference sequences used are NG_016311.1 and NM_177438.2. Patient and mutation details are available in the DICER1 Leiden Open Variant Database (https://grenada.lumc.nl/LOVD2/mendelian_genes/home.php?select_db=DICER1).

\section{RESULTS AND DISCUSSION}

In individual iii-1 (Figure 1 and last row Table 1), we identified a germ-line deletion of exon 21 in DICER1, c.3270-6_4051-1280delinsG (Figure $2 \mathrm{a}-\mathrm{c}$ ). The deletion was also present in her mother (ii-2 and Supplementary Figure S2 (1-A and 1-B)) and brother (iii-2 and 
Supplementary Figure S2 (2-D and 2-E)). Exon 21 spans from c.3270 to c.4050, which corresponds to the amino-acid positions 1031-1349. The RNase IIIa domain located between amino-acid 1296 and 1376 in the human DICER1 protein ${ }^{14}$ is critical for the production of miRNAs from the $3^{\prime}(3 \mathrm{p})$ arm of the precursors. ${ }^{15}$ The heterozygous deletion of exon 21 eliminates a region of the gene that encodes a large part of RNase IIIa. It disrupts the last codon in exon 20 by removing the first nucleotide of exon 21, which creates a frameshift predicted to truncate the protein (p.Tyr1091Ser ${ }^{\star} 28$ ). The genomic deletion is 2918 base pairs in length (Figure 2d). Using long-range PCR, we were able to identify the breakpoints of the deletion: the $5^{\prime}$ end is located in intron 20 (c.3270-6delttgcag); six base pairs from the start of exon 21 are deleted along with exon 21 (781 base pairs). The deletion terminates at a TTGCAGC sequence in the middle of intron 21 (c.4051-1280), followed by an insertion of a $G$ nucleotide and results in g.58291_61208delinsG (Figure 2e1 and e2). Chromatograms illustrating this are shown in Figure $2 \mathrm{f}$ and g. Sequencing of cDNA revealed the absence of exon 21 (r.3270_4050del) from the proband's lymphocyte RNA (Figure $2 \mathrm{~h}$ ) and confirmed that the mRNA is not subject to nonsense-mediated decay.

The combination of SLCTs and familial MNG is particularly suggestive of a germ-line DICER1 mutation, ${ }^{5}$ and therefore the lack of a mutation in DICER1 by conventional Sanger sequencing in this patient and similar others shown in Table 1 prompted us to develop the DICER1 MLPA assay described here. As a result, we identified this novel disease-causing large deletion. It is likely that a further search into the $5^{\prime}$ and $3^{\prime}$ UTRs of DICER1 will reveal other disease-associated mutations in some of the families listed in Table 1. It is possible, however, that other members of the miRNA processing pathway, such as DROSHA which, like DICER1, possesses conserved RNase III domains, ${ }^{16}$ will be found to carry deleterious mutations. Mutations in other genes that encode miRNA processors such as DGCR8 and Argonaute might also result in altered miRNA profiles that seem to be a hallmark of diseaseassociated mutations in DICER1 mutations. ${ }^{5,15,17,18}$

MLPA has proved to be a very useful method for detecting deletions, duplications and rearrangements in genes. We estimate that between 1 and $10 \%$ of all germ-line mutations in DICER 1 will be detectable by MLPA but will be missed by conventional Sanger sequencing. For this reason, it will be important to include MLPA, or related methods, in the assessment of those suspected of carrying germ-line DICER1 mutations.

\section{CONFLICT OF INTEREST}

The authors declare no conflict of interest.

\section{ACKNOWLEDGEMENTS}

We thank Dr John R Priest for his continued support of our work and all those who provided samples, especially Tomasz Dzik, MD, Ph.D. We would like to thank Jonathan Jarry, M.Sc. and George Chong, Ph.D. for providing input and help with the implementation of the MLPA assay. We also thank the technical support team from PREMIER Biosoft and Jelger van der Meer Ph.D.
(MRC-Holland) for advice in devising the probe-mixes and MLPA assays, respectively. The study was financially supported by the Mendon F Schutt Foundation and the Jewish General Hospital Foundation.

\section{AUTHOR CONTRIBUTIONS}

NS developed the MLPA assay, performed the validation in genomic DNA and wrote the manuscript; AS prepared the RNA and cDNA and performed the cDNA experiments; $\mathrm{NH}$ oversaw the laboratory work and edited the manuscript; FP helped to recruit the cases; MM and $\mathrm{MN}$ provided samples and key clinical information; WDF wrote the paper with NS and led the project. All authors read and approved the final submitted manuscript.

1 Hill DA, Ivanovich J, Priest JR et al: DICER1 mutations in familial pleuropulmonary blastoma. Science 2009; 325: 965.

2 Priest JR, McDermott MB, Bhatia S, Watterson J, Manivel JC, Dehner LP: Pleuropulmonary blastoma: a clinicopathologic study of 50 cases. Cancer 1997; 80: 147-161.

3 Bahubeshi A, Bal N, Frio TR et al: Germ-line DICER1 mutations and familial cystic nephroma. J Med Genet 2010; 47: 863-866.

4 Slade I, Bacchelli C, Davies H et al: DICER1 syndrome: clarifying the diagnosis, clinical features and management implications of a pleiotropic tumour predisposition syndrome. J Med Genet 2011; 48: 273-278.

5 Rio Frio T, Bahubeshi A, Kanellopoulou C et al: DICER1 mutations in familial multinodular goiter with and without ovarian Sertoli-Leydig cell tumors. JAMA 2011; 305: 68-77.

6 Foulkes WD, Bahubeshi A, Hamel N et al: Extending the phenotypes associated with DICER1 mutations. Hum Mutat 2011; 32: 1381-1384.

7 Sabbaghian N, Hamel N, Srivastava A, Albrecht S, Priest JR, Foulkes WD: Germ-line DICER 1 mutation and associated loss of heterozygosity in a pineoblastoma. J Med Genet 2012; 49: 417-419.

8 Wildi-Runge S, Bahubeshi A, Carret $\mathrm{A}$ et al: New phenotype in the familial DICER1 tumor syndrome: pituitary blastoma presenting at age 9 months. Endocr Rev 2011; 32: $\mathrm{P} 1-777$.

9 Hogervorst FB, Nederlof PM, Gille JJ et al: Large genomic deletions and duplications in the BRCA1 gene identified by a novel quantitative method. Cancer Res 2003; 63 . 1449-1453.

10 Stuppia L, Antonucci I, Palka G, Gatta V: Use of the MLPA assay in the molecular diagnosis of gene copy number alterations in human genetic diseases. Int $\mathrm{J} \mathrm{Mol} \mathrm{Sci}$ 2012; 13: 3245-3276.

11 Chong G, Jarry J, Marcus V et al: High frequency of exon deletions and putative founder effects in French Canadian Lynch syndrome families. Hum Mutat 2009; 30: E797-E812.

12 MRC-Holland b.v. Designing synthetic MLPA probes. Version 10. 4-2-2009.

13 Allele ID v7.7 2013; http://www. premierbiosoft.com/bacterial-identification/realtime$\mathrm{PCR} / \mathrm{species-identification.html}$

14 Blaszczyk J, Tropea JE, Bubunenko M et al: Crystallographic and modeling studies of RNase III suggest a mechanism for double-stranded RNA cleavage. Structure 2001; 9: 1225-1236.

15 Gurtan AM, Lu V, Bhutkar A, Sharp PA: In vivo structure-function analysis of human Dicer reveals directional processing of precursor miRNAs. RNA 2012; 18: 1116-1122.

16 Nowotny M, Yang W: Structural and functional modules in RNA interference. Curr Opin Struct Biol 2009; 19: 286-293.

17 Heravi-Moussavi A, Anglesio MS, Cheng SW et al: Recurrent somatic DICER1 mutations in nonepithelial ovarian cancers. N Engl J Med 2012; 366: 234-242.

18 Anglesio M, Wang Y, Yang W et al: Cancer-associated somatic DICER1 hotspot mutations cause defective miRNA processing and reverse strand expression bias to predominantly mature $3 p$ strands through loss of $5 p$ strand cleavage. J Pathol 2013; 229: 400-409.

Supplementary Information accompanies this paper on European Journal of Human Genetics website (http://www.nature.com/ejhg) 